OPEN ACCESS

Edited by:

Shawn Hervey-Jumper University of California, San Francisco,

United States

Reviewed by:

Alfredo Conti,

Università degli Studi di Messina, Italy

Martin Rutkowski,

University of Southern California,

United States

*Correspondence:

Sandro M. Krieg

Sandro.Krieg@tum.de

Specialty section:

This article was submitted to

Neuro-Oncology and Neurosurgical

Oncology,

a section of the journal

Frontiers in Oncology

Received: 19 September 2018

Accepted: 15 November 2018

Published: 06 December 2018

Citation:

Ille S, Engel L, Kelm A, Meyer B and Krieg SM (2018) Language-Eloquent

White Matter Pathway Tractography and the Course of Language Function

in Glioma Patients.

Front. Oncol. 8:572.

doi: 10.3389/fonc.2018.00572

\section{Language-Eloquent White Matter Pathway Tractography and the Course of Language Function in Glioma Patients}

\author{
Sebastian IIle ${ }^{1,2}$, Lara Engel ${ }^{1,2}$, Anna Kelm ${ }^{1,2}$, Bernhard Meyer ${ }^{1}$ and Sandro M. Krieg ${ }^{1,2 *}$ \\ ${ }^{1}$ Department of Neurosurgery, Klinikum Rechts der Isar, Technische Universität München, Münich, Germany, \\ ${ }^{2}$ TUM-Neuroimaging Center, Klinikum Rechts der Isar, Technische Universität München, Münich, Germany
}

Object: As various recent studies show, damage to white matter pathways leads to permanent functional deficits in a high percentage of patients. Particularly the subcortical language network is complex, and its visualization has a tremendous relevance for neurosurgeons. This pilot study aims to correlate language-eloquent white matter pathways with the course of language function after the resection of left-sided perisylvian gliomas.

Methods: We included 10 patients who underwent resection of highly language-eloquent high- (9 pts) and low-grade gliomas (1 pts). We performed navigated repetitive transcranial magnetic stimulation (nrTMS)-based tractography via diffusion tensor imaging fiber trackings (DTI FT) preoperatively (PRE-1), postoperatively (POST-1), and at long-term follow up or tumor recurrence (PRE-2). We separately tracked the inferior fronto-occipital fascicle (IFOF), the frontal aslant tract (FAT), and the superior longitudinal (SLF), and arcuate fascicle (AF), and correlated the amount of visualized fibers to the patients' language function at each date.

Results: The changes of nrTMS-based DTI FTs of single white matter pathways correlated with the according status of language function for any of the pathways in $80 \%$ of patients and in 19 of 30 (63\%) single pathway comparisons between PRE-1 and POST-1. Between POST-1 and PRE-2 the nrTMS-based DTI FTs correlated with the status of language function for any of the pathways in all patients and in 24 of 30 (80\%) single pathway comparisons. Single FT results correlated with the according status of language function at POST-1 in 60, 70, and $60 \%$ of cases, and with the according status of language function at PRE-2 in 60,90 , and $90 \%$ of cases for the tracking of the IFOF, FAT, and SLF/AF, respectively.

Conclusion: By the present results we were able to show that nrTMS-based DTI FT of the IFOF, FAT, and SLF/AF mainly correlates with the according status of language function preoperatively, postoperatively, and at long-term follow up after the resection of left-sided perisylvian gliomas.

Keywords: fiber tracking, glioma, language, nrTMS, tractography 


\section{INTRODUCTION}

Cortical and subcortical anatomy of human language function is complex. Decades after Broca, Wernicke, and Geschwind we know that cortical language function is highly individualized and only the superficial part of a complex network. As we have learned from studies examining cortical and subcortical representations of language function during the resection of language-eloquent gliomas by the gold standard technique direct electrical stimulation (DES) during awake surgery, large parts of the cortex are resectable without causing a functional deficit, while subcortical white matter pathways need to be preserved to a larger extent (1-3). Hence, preserving these structures is essential during the resection of eloquent brain tumors. Our current knowledge of the complex subcortical language network bases on white matter tractography by diffusion tensor imaging fiber tracking (DTI FT) and insights through DES during awake craniotomies (4-8). These techniques and anatomical studies led to the currently most accepted dual stream model of language $(8,9)$. The ventral stream passing through the external capsule (EC) and consisting of the inferior frontooccipital fascicle (IFOF), the uncinate fascicle (UF), and the inferior longitudinal fascicle (ILF) carries semantic information and connects associated cortical areas of the frontal, temporal, parietal, and occipital lobe (8-10). The dorsal stream, which runs around the sylvian fissure connecting the frontal, temporal, and parietal perisylvian cortices, includes the arcuate fascicle (AF) and parts of the superior longitudinal fascicle (SLF) and transmits phonological processing and articulation $(8,9)$. Apart from this long-connectivity language network, recently published studies confirmed the language-eloquent role of the frontal aslant tract (FAT) connecting the supplemental motor areas (SMA) and pre-SMA with Broca’s region (11-14).

Meanwhile, language mapping by navigated repetitive transcranial magnetic stimulation (nrTMS) has emerged to a commonly used preoperative mapping technique (15-18). It enables to perform a cortical mapping by inducing virtual lesions non-invasively and is thereby based on the same principle of functioning as the gold standard technique DES during awake craniotomy (19). Also the feasibility of visualizing subcortical white matter pathways by nrTMS-based DTI FT has repeatedly been shown $(20,21)$. Hence, the approach of choosing function-based regions of interest (ROI) in terms of a cortical nrTMS language mapping enables to select single white matter pathway tractographies. Additionally, the non-invasive mapping technique provides the opportunity to perform function-based DTI FTs during long-term follow up without tumor recurrence.

\footnotetext{
Abbreviations: AAT, Aachener Aphasia Test; AF, Arcuate fascicle; DES, Direct electrical stimulation; DTI FT, Diffusion tensor imaging fiber tracking; EC, External capsule; FAT, Frontal aslant tract; FACT, Fiber assignment by continuous tracking; IFOF, Inferior fronto-occipital fascicle; ILF, Inferior longitudinal fascicle; nrTMS, Navigated repetitive transcranial magnetic stimulation; ON, Object naming task; POD5-1/-2, 5 days after surgery $1 / 2$; POM3-1/-2, 3 months after surgery 1/2; POST-1, Between PRE-1 and PRE-2; PRE-1/-2, Before surgery 1/2; ROI, Region of interest; SLF, Superior longitudinal fascicle; SMA, Supplementary motor areas; UF, Uncinate fascicle.
}

The present study evaluates if we are able to perform single white matter pathway tractographies of the FAT, the IFOF, and the SLF/AF as the core of white matter language pathways by nrTMS-based DTI FT in patients suffering from highly languageeloquent gliomas. Furthermore, the study examines if we can visualize the changes of the extension of the subcortical language network in dependence on the status of language function preoperatively, postoperatively, and at long-term follow up.

\section{MATERIALS AND METHODS \\ Ethics}

The experimental setup was approved by our local ethics committee (registration number: 222/14) and was conducted in accordance with the Declaration of Helsinki. Written informed consent was obtained from all patients prior to the examination.

\section{Patients}

For the present study we included patients with left-sided language-eloquent gliomas of our prospective language mapping cohort. In order to perform nrTMS-based DTI FT preoperatively, postoperatively, and at long-term follow up, patients had to meet the inclusion criteria of repeated nrTMS language mappings and DTI sequences. Patients with an age younger than 18 years, general TMS exclusion criteria, such as cochlear implants or a cardiac pacemaker, or a too severe aphasia $(<60 \%$ properly named pictures during baseline object naming) were excluded from the study $(22,23)$.

\section{Language Assessment}

For the present analysis we evaluated the patients' neurological statuses including an aphasia grading adapted from the Aachener Aphasia Test (AAT) (24) at no fewer than six points in time: before surgery 1 (PRE-1), 5 days after surgery 1 (POD5-1), 3 months after surgery 1 (POM3-1), before surgery 2 or during long-term standard follow-up in case of no tumor recurrence (PRE-2), 5 days after surgery 2 (POD5-2), and 3 months after surgery 2 (POM3-2). We graded aphasias from 0 to $3(0=$ no impairment of language function; $1=$ slight impairment of daily communication; $2=$ moderate impairment of language function, daily communication possible; $3=$ severe impairment of language function, daily communication not possible), and in case of an aphasia by the addition of an A (non-fluent) or B (fluent) $(15-18,20,24,25)$.

\section{Magnetic Resonance Imaging}

The sequences were performed on $3 \mathrm{~T}$ magnetic resonance scanners (Philips Medical System, Netherlands B.V.). All patients obtained MRIs according to the standard glioma protocol at our department including a T1-weighted three-dimensional (3-D) gradient echo sequence with intravenous contrast administration for anatomical co-registration, a T2-weighted 3-D FLAIR sequence, and DTI sequences with 6-32 orthogonal sequences. T1- and T2-weighted 3-D images were performed at each of the six points in time at which language performance was evaluated. We used the postoperative diffusion images at POD5-1 to evaluate subcortical ischemia. DTI sequences 
for nrTMS-based DTI FTs were conducted at PRE-1, PRE2 , and at one point in time between PRE-1 and PRE-2 (POST-1).

\section{Operative Technique}

All surgeries were performed with a function-based approach for the resection of language-eloquent gliomas. Standardly, we used a neuronavigation system for all surgeries (Brainlab Curve, Brainlab AG, Munich, Germany). The neuronavigation system was used for craniotomy planning and intraoperative navigation. Additionally, preoperative nrTMS language mapping results and nrTMS-based DTI FTs were transferred to the neuronavigation system in all cases and thereby displayed on the navigation screen during the microsurgical resection. In cases of an additional language mapping by DES during awake craniotomy, the intraoperative mapping was guided by preoperative nrTMS language mapping data (26).

Within the evaluated period we performed eight language mappings by DES during awake craniotomy in the included patients. We therefore used our standard protocol with an asleep-awake-asleep approach and according to the guidelines for awake craniotomies (27-29). In these cases we used a combination of epinephrine and bupivacaine for regional anesthesia of the galea and dura, and a total intravenous anesthesia by remifentanil and propofol, which was stopped prior to intraoperative language mapping. Here, we performed an object naming task $(\mathrm{ON})$ consisting of the pictures which were used for the ON during preoperative nrTMS language mapping. In order to detect intraoperative seizures we recorded a surface electroencephalogram. Cortical stimulation was performed with a bipolar electrode and subcortical stimulation was performed with a monopolar electrode (Inomed Medizintechnik $\mathrm{GmbH}$, Emmendingen, Germany). After the language mapping phase the resection was performed under continuous monitoring of overt speech.

\section{Setup}

nrTMS Language Mapping and nrTMS-Based DTI FT

We performed nrTMS language mappings using the eXimia nTMS system version 4.3 and a NEXSPEECH ${ }^{\circledR}$ module (Nexstim Plc, Helsinki, Finland) and an ON at PRE-1 and PRE-2 according to the recently published nTMS working group protocol (23). Afterwards, we exported the left-sided language-positive sites in terms of nrTMS in order to perform nrTMS-based DTI FT.

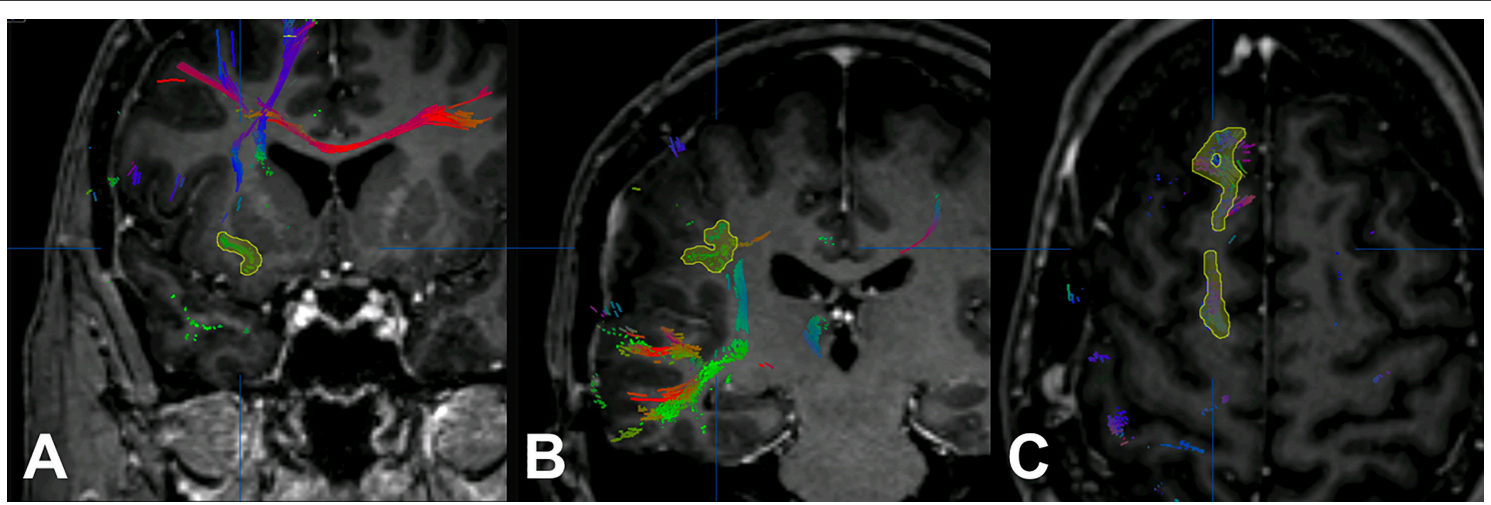

FIGURE 1 | Additional regions of interest. The figure shows the process of adding further regions of interest for the inferior fronto-occipital fascicle (IFOF; A), the superior longitudinal fascicle and arcuate fascicle (SLF/AF; B), and the frontal aslant tract (FAT; C).

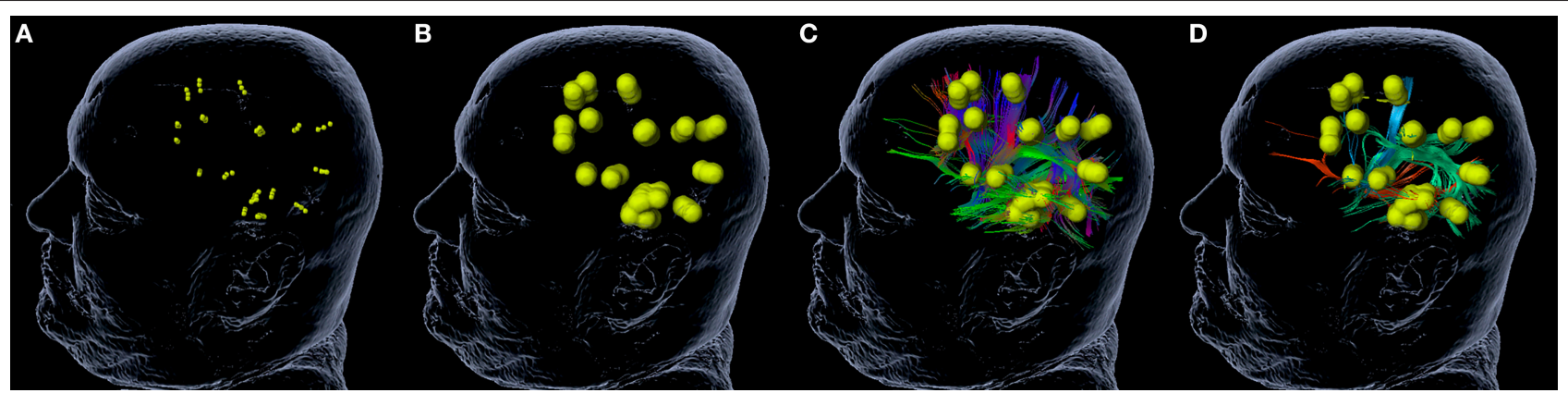

FIGURE 2 | Tractography of single white matter pathways. The figure shows the process of tractography for single white matter pathways. A rim of 5 mm (B) is added to language-positive sites in terms of nrTMS (A). The software calculates a whole-language network tractography (C). By the addition of further regions of interest (ROI, Figure 1) the whole-language network tractography can be reduced to the inferior fronto-occipital fascicle (IFOF; red), the superior longitudinal fascicle and arcuate fascicle (SLF/AF; green), and the frontal aslant tract (FAT; blue) (D). 
For nrTMS-based DTI FT of language-eloquent white matter pathways we used our standard deterministic algorithm with a fiber assignment by continuous tracking (FACT) (iPlanNet Cranial 3.0.1, Brainlab AG, Munich, Germany). The DTI FTs were performed according to our standard protocol $(18,20,25)$. The DTI FTs at PRE- 1 and PRE-2 were performed by the use of nrTMS language mapping results and the according DTI sequences at PRE- 1 and PRE-2. The DTI FT at POST- 1 was performed by the use of nrTMS language mapping results at PRE- 1 and the DTI sequences at POST-1. First, we performed a FT of all subcortical pathways connecting language-positive cortical sites in terms of nrTMS (Figure 1). Based on the resulting whole-language-network tractography we added further ROIs in order to track single pathways. For the tracking of the IFOF we set an additional ROI within dorso-rostrally oriented fibers of the external capsule. The SLF/AF was tracked by adding an additional ROI within dorso-rostrally oriented fibers lateral to the posterior horn of the lateral ventricle. For the tracking of the FAT we set an additional ROI within cranio-caudally oriented fibers connecting the superior frontal gyrus and the inferior frontal gyrus (Figure 2). Thereby, we were able to separately visualize single pathways, which were exclusively connecting languagepositive cortical sites in terms of nrTMS and additionally passing through one of the three additional ROIs (Figures 1, 2).

\section{Data Analysis}

In order to correlate the FT results at PRE-1, POST-1, and PRE-2 with the according status of language function, we analyzed the number of fibers for the purpose of a volumetric analysis as calculated by the fiber tracking software (iPlanNet Cranial 3.0.1, Brainlab AG, Munich, Germany). Therefore, we calculated the absolute and relative differences for each pathway at the three examinations. This was done separately for all patients and summarized for the following groups: patients with permanent surgery-related language deficits (= new postoperative language deficit at POD5-1 and POM3-1), patients with transient surgery-related language deficits $(=$ new postoperative language deficit at POD5-1, no language deficit at POM3-1), patients with new surgery-related language deficits, patients with new tumor-related language deficits (= no language deficit at POM3-1, new language deficit at PRE-2 and tumor recurrence in MRI scan), and patients without new language deficits.

\section{RESULTS}

\section{Patient and Tumor Characteristics}

We included 10 patients with a mean age of $50 \pm 13.9$ years. Table 1 shows detailed patient characteristics of all included patients including the intervals between the different MR images, the extent of resection (EOR), and the detection of subcortical ischemia after the first surgery. Furthermore, Table 1 gives information about the status of language function at each examination during the evaluation period and the assignment of each patient to the different subgroups "permanent surgeryrelated language deficits," "transient surgery-related language

TABLE 1 | Patient characteristics.

\begin{tabular}{|c|c|c|c|c|c|c|c|c|}
\hline Patient & Age & $\begin{array}{l}\text { PRE-1 to } \\
\text { PRE-2 } \\
\text { (months) }\end{array}$ & $\begin{array}{l}\text { PRE-1 to } \\
\text { 2nd DTI } \\
\text { (days) }\end{array}$ & $\begin{array}{l}\text { PRE-1 to } \\
\text { 3rd DTI } \\
\text { (months) }\end{array}$ & $\begin{array}{l}\text { Subcortical } \\
\text { ischemia after } \\
\text { surgery } 1\end{array}$ & EOR & Aphasia grading & $\begin{array}{l}\text { Course of } \\
\text { language function }\end{array}$ \\
\hline
\end{tabular}

PRE-1 POD5-1 POM3-1 PRE-2 POD5-2 POM3-2

\begin{tabular}{|c|c|c|c|c|c|c|c|c|c|c|c|c|c|}
\hline 1 & 45 & 12 & 15 & 6 & $\mathrm{~N}$ & GTR & 0 & $1 \mathrm{~A}$ & $1 \mathrm{~A}$ & $1 \mathrm{~A}$ & - & - & $\begin{array}{l}\text { Permanent } \\
\text { surgery-related } \\
\text { deficit }\end{array}$ \\
\hline 2 & 34 & 26 & 7 & 26 & $\mathrm{Y}$ & GTR & 0 & $1 \mathrm{~A}$ & 0 & 0 & 0 & 0 & $\begin{array}{l}\text { Transient } \\
\text { surgery-related } \\
\text { deficit }\end{array}$ \\
\hline 3 & 49 & 37 & 189 & 25 & Y & GTR & 0 & $1 \mathrm{~B}$ & 0 & 0 & - & - & $\begin{array}{l}\text { Transient } \\
\text { surgery-related } \\
\text { deficit }\end{array}$ \\
\hline 4 & 74 & 2 & 57 & 2 & $N$ & GTR & 0 & $2 \mathrm{~A}$ & 0 & 0 & - & - & $\begin{array}{l}\text { Transient } \\
\text { surgery-related } \\
\text { deficit }\end{array}$ \\
\hline 5 & 31 & 15 & 1 & 14 & $\mathrm{~N}$ & GTR & $1 \mathrm{~B}$ & $1 \mathrm{~B}$ & 0 & $1 \mathrm{~A}$ & $1 \mathrm{~A}$ & $1 \mathrm{~A}$ & $\begin{array}{l}\text { New tumor-related } \\
\text { deficit }\end{array}$ \\
\hline 6 & 51 & 14 & 3 & 14 & $\mathrm{~N}$ & GTR & 0 & 0 & 0 & 1B & $1 \mathrm{~B}$ & $1 \mathrm{~B}$ & $\begin{array}{l}\text { New tumor-related } \\
\text { deficit }\end{array}$ \\
\hline 7 & 56 & 3 & 5 & 3 & $\mathrm{~N}$ & GTR & 0 & 0 & 0 & $1 \mathrm{~A}$ & $2 \mathrm{~A}$ & $1 \mathrm{~A}$ & $\begin{array}{l}\text { New tumor-related } \\
\text { deficit }\end{array}$ \\
\hline 8 & 52 & 21 & 120 & 22 & $\mathrm{~N}$ & GTR & $2 \mathrm{~B}$ & $1 \mathrm{~B}$ & 0 & 0 & 0 & $2 \mathrm{~A}$ & Without new deficit \\
\hline 9 & 36 & 30 & 13 & 28 & $\mathrm{~N}$ & GTR & 0 & 0 & 0 & 0 & - & - & Without new deficit \\
\hline 10 & 72 & 13 & 7 & 13 & $\mathrm{~N}$ & GTR & 0 & 0 & 0 & 0 & 0 & 0 & Without new deficit \\
\hline
\end{tabular}

The table shows detailed patient characteristics of all included patients including the intervals between the different MR images, the status of language function at each examination, and the assignment of patients to different subgroups (Y, yes, N, no; EOR, extend of resection, GTR, gross total resection). 
deficits," "new tumor-related language deficits," and "without new language deficit" (Table 1).

Table 2 shows detailed information about the tumor type of each patient including WHO grading, IDH mutation, and $1 \mathrm{p} 19 \mathrm{q}$ codeletion. Moreover, the table gives information regarding the location of the tumor and if more than $50 \%$ of the tumor volume were located subcortically (Table 2).

\section{Fiber Tracking Analysis}

\section{Single White Matter Pathway Analysis}

Table 3 shows the absolute number of fibers for each of the separately tracked subcortical pathways at PRE-1, and the percentage changes of nrTMS-based DTI FTs between PRE1 and POST-1, POST-1, and PRE-2, and PRE-1, and PRE-2 (Table 3). The changes of nrTMS-based DTI FTs of single white matter pathways correlated with the according status of language function for any of the pathways in $80 \%$ of patients and in 19 of $30(63 \%)$ single pathway comparisons between PRE-1 and POST-1. Between POST-1 and PRE-2 the nrTMS-based DTI FTs correlated with the status of language function for any of the pathways in all patients and in 24 of 30 (80\%) single pathway comparisons. Single FT results correlated with the according status of language function at POST-1 in $60 \%, 70 \%$, and $60 \%$ of cases, and with the according status of language function at PRE2 in $60 \%, 90 \%$, and $90 \%$ of cases for the tracking of the IFOF, FAT, and SLF/AF, respectively (Table 3 ).

\section{Subgroup Analysis}

Postoperatively, we found an overall loss of fibers of the IFOF, FAT, and SLF/AF of $-79.8 \%$ between PRE- 1 and POST-1 in patients suffering from new surgery-related language deficits. Three patients with transient surgery-related language deficits appropriately showed an overall gain of fibers of $48.4 \%$ between POST-1 and PRE-2. In contrast, one patient suffering from a permanent surgery-related language deficit also showed an overall gain of fibers of $49.4 \%$ depending on a distinct gain of fibers within the IFOF, while fibers within the FAT and the
SLF/AF stayed low (Figure 3). Patients with new tumor-related language deficits at PRE-2 showed an overall loss of fibers of $-75.5 \%$ between POST-1 and PRE-2 (Figure 4). These patients also showed an overall loss of fibers between PRE-1 and POST-1. Three patients without new language deficits showed an overall gain of fibers of $124.8 \%$ between PRE- 1 and POST-1, and an overall gain of fibers of $27.8 \%$ between POST-1 and PRE-2 (Table 4).

\section{DISCUSSION}

\section{Correlation of Language Pathways With the Course of Language Function}

By the present results we were able to show that nrTMSbased DTI FT of the IFOF, FAT, and SLF/AF mainly correlates with the according status of language function preoperatively, postoperatively, and at long-term follow up. Especially new language deficits could be visualized with a high reliability (Figures 3, 4 and Tables 3, 4). In these cases, we could also show a high correlation of the kind of language deficit and the according pathway. In patients suffering from new non-fluent aphasias, we found a loss of SLF/AF and FAT fibers, which are both responsible for phonological processing, articulation, and articulatory planning (Figure 3). In contrast, patients with new fluent aphasias showed a distinct loss of IFOF fibers, which are responsible for semantic information (Tables $\mathbf{1}, \mathbf{3})(8,9)$.

The tractography of single white matter pathways particularly correlated for the comparisons between POST-1 and PRE-2. In patients suffering from new surgery-related language deficits we could find a loss of fibers at POST- 1 and a gain of fibers at PRE2 in case of transient language deficits. Although we found an overall gain of fibers for the comparison between POST-1 and PRE-2 in one patient suffering from a permanent language deficit, we still could find less fibers at PRE-2 in comparison with PRE1 (Figure 3, Table 3). The total gain of fibers at PRE-2 in this patient was due to an increase of IFOF fibers while FAT and

TABLE 2 | Tumor characteristics.

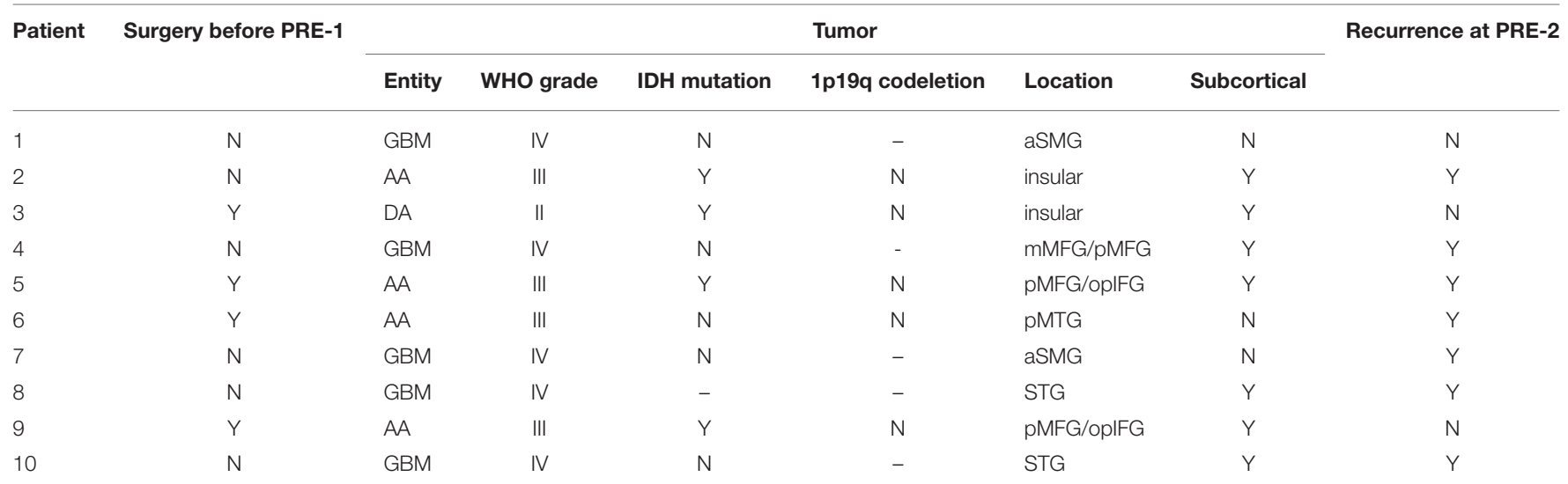

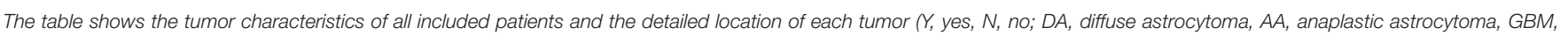

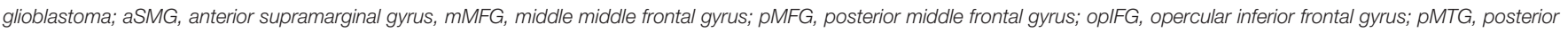
middle temporal gyrus; STG, superior temporal gyrus). 


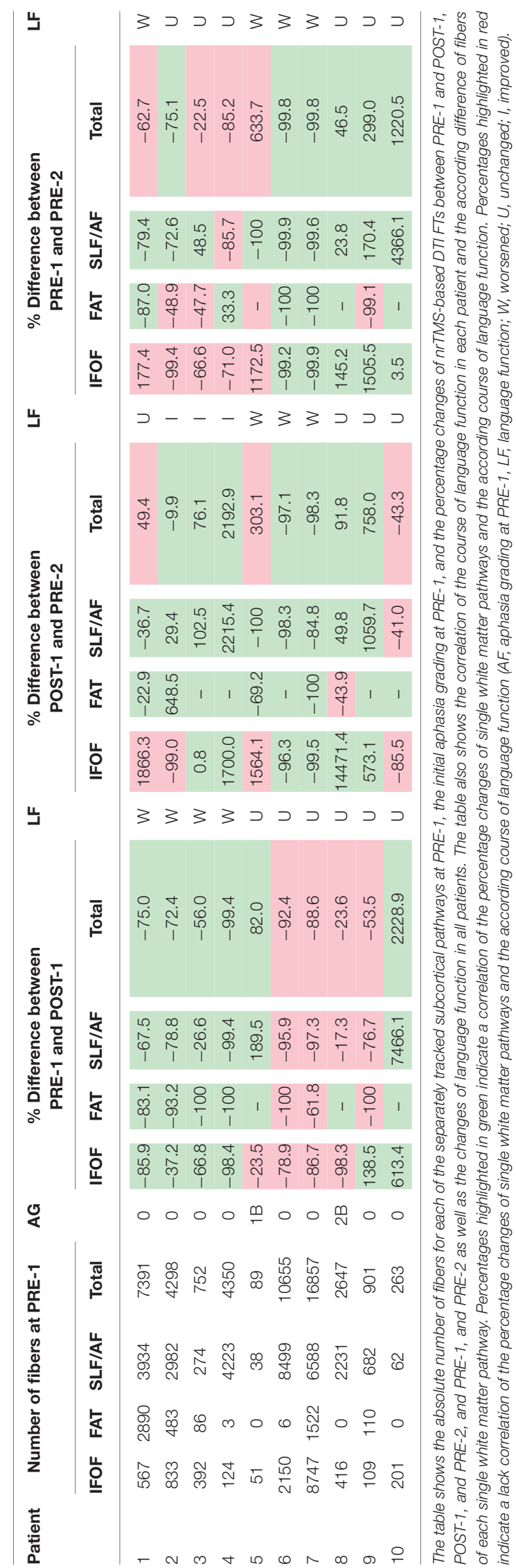

SLF/AF fibers were still decreasing. Conveniently, this patient suffered from a permanent non-fluent language deficit. Again, the tractography result of single tracts correlated well with the corresponding language function and kind of deficit as described in literature $(9,30)$. Patients with new tumor-related language deficits showed an overall loss of fibers at PRE-2, however, these patients already showed an overall loss of fibers at POST-1. In contrast, we could not find a loss of fibers at POST- 1 or PRE- 2 in patients without any language deficit (Table 4).

\section{Structural Lesions and Compensatory Mechanisms}

As we have learned from DES studies providing resection probability maps, large parts of the cortex are resectable, but lesions within the subcortical white matter pathways cause permanent language deficits in most cases $(2,3)$. However, this fact does not rule out the possibility of functional reorganization and compensatory mechanisms on a subcortical level. That functional reorganization is able to compensate functional deficits cortically by various mechanisms has already been shown by former studies $(16,30-32)$. Hereby, the auto-regulation of synapses and mechanisms for the unmasking of latent networks play a major role (33-35). That the human subcortical white matter per se harbors multipotential neural progenitor cells has already been proven (36). However, we cannot be sure whether damage to white matter pathways leads to functional reorganization on a subcortical level by rewiring, or whether subcortical changes are only a passive reaction on cortical mechanisms. Not least the results of the present study show that the whole network is able to compensate lesions by the recruitment of accessory and parallel long-distance association pathways and the unmasking of perilesional latent parallel networks (37). These mechanisms might be a reason for the appropriate visualization of a gain of fibers between POST-1 and PRE-2 in patients suffering from transient surgery-related language deficits.

Nevertheless, the impact of surgically induced deficits happens to fast for compensatory mechanisms. Our cohort includes four patients with new surgery-related language deficits and we could find a loss of fibers between PRE- 1 and POST-1 for each of the visualized pathways and in all of these 4 patients. However, we could find subcortical ischemia in postoperative MR images in only two cases. Thus, damage to white matter pathways seems not to be an essential reason for subcortical changes. These changes might originate from the cortex. Since these two patients suffered from transient language deficits, the recovery of unimpaired language function as measured at POM3-1 might be due to functional reorganization on the subcortical level. In two patients with new surgery-related language deficits without subcortical ischemia we were also able to find a loss of fibers. In these cases, the loss of fibers must have been caused by the loss of connectivity without a structural lesion on the subcortical level. This hypothesis is supported by the fact that we used the same ROIs of language-positive cortical sites in terms of nrTMS at PRE-1 and POST-1. 


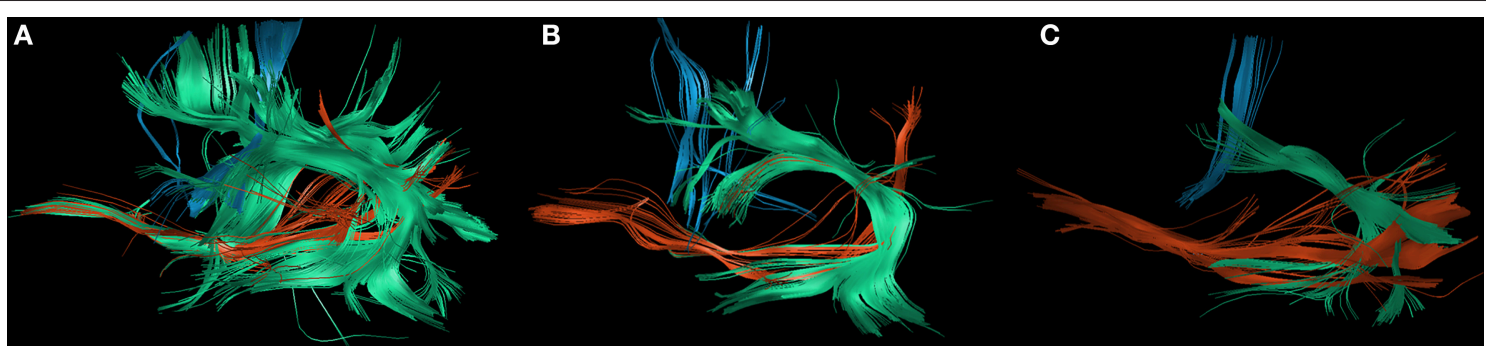

FIGURE 3 | Changes of white matter pathways after permanent surgery-related deficit. The figure shows the changes of the inferior fronto-occipital fascicle (IFOF; red), the superior longitudinal fascicle and arcuate fascicle (SLF/AF; green), and the frontal aslant tract (FAT; blue) at PRE-1 (A), POST-1 (B), and PRE-2 (C) in patient 1 who suffered from a permanent surgery-related language deficit grade $1 \mathrm{~A}$.

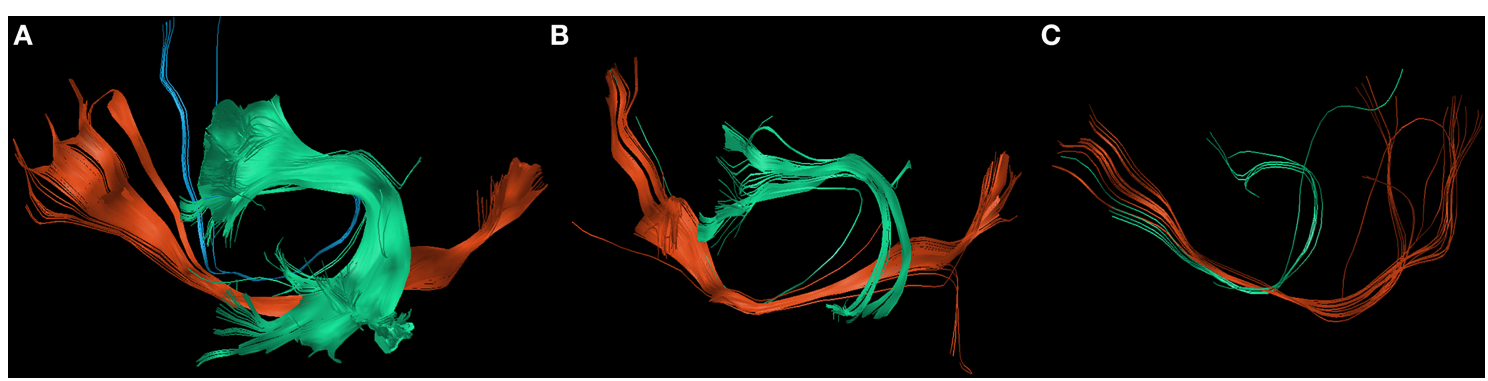

FIGURE 4 | Changes of white matter pathways after tumor-related deficit. The figure shows the changes of the inferior fronto-occipital fascicle (IFOF; red), the superior longitudinal fascicle and arcuate fascicle (SLF/AF; green), and the frontal aslant tract (FAT; blue) at PRE-1 (A), POST-1 (B), and PRE-2 (C) in patient 6 who suffered from a new tumor-related language deficit grade 1B.

We were also able to measure a loss of fibers in 3 patients suffering from new tumor-related language deficits at PRE-2. In contrast, we could not find a loss of fibers in another four patients who also showed a tumor recurrence at PRE-2 without causing a language deficit. Thereby, in case of tumor-related language deficits it has to be assumed that the lack of fibers is caused by an impairment of the whole network. Here, compensatory mechanisms on a cortical and subcortical level seem to be successful regarding the preservation of language function only in some cases. Furthermore, patients with transient surgeryrelated language deficits after the improvement of their function and patients without any deterioration of language function after the resection of the compromising glioma even showed a gain of fibers.

\section{Factors Impairing the Reliability of DTI FT}

In the present analysis the comparisons of single white matter language pathways correlated with the according status of language function in $63 \%$ at POST-1 and $80 \%$ at PRE-2, respectively. When analyzing the mismatch of fibers and the status of language function more detailed, we can disregard the mismatch of IFOF fibers at PRE-2 in case 1, 2, and 5. These patients suffered from non-fluent aphasias but IFOF fibers transfer semantic information predominantly. After this correction the mismatch of fibers and status of language function at PRE- 2 is only $10 \%$.
In contrast, the reliability of nrTMS-based DTI FT seems to be more impaired directly after surgery. In patients 5, 6, and 7 , who did not suffer from a new language deficit at POST1 , we found the highest mismatch of fibers and the according status of language function. Characteristically, these patients had the shortest intervals between PRE-1 and the second DTI imaging at POST-1. Hence, it must be assumed that postoperative edema impairs DTI imaging, even if it does not cause a language deficit. Furthermore, this hypothesis is supported by the results of patients 1-4. These patients suffered from new surgery-related language deficits and their status of language function correlated with nrTMS-based DTI FT results in all cases and for each white matter pathway. Significantly, the intervals between PRE-1 and POST-1 were longer in these cases.

\section{Future Applications of the Presented Approach}

For the present study we used DTI FT as a research tool. We also perform preoperative nrTMS-based DTI FT for motor and language pathways standardly at our department and transfer these data to neuronavigation for the intraoperative illustration (38). Although DTI FT has repeatedly been compared to the gold standard technique of intraoperative subcortical stimulation showing promising results $(4,39-41)$, it might still serve as a research tool and not a clinical tool (42). In order to preserve function during subcortical glioma resection, the limitations of the underlying techniques per se have to be kept in mind. These 
TABLE 4 | Subgroup analysis.

\begin{tabular}{|c|c|c|c|c|}
\hline & IFOF & FAT & SLF/AF & Total \\
\hline \multicolumn{5}{|c|}{ DIFFERENCE BETWEEN PRE-1 AND POST-1 IN NEW } \\
\hline \multicolumn{5}{|c|}{ SURGERY-RELATED LANGUAGE DEFICITS } \\
\hline Relative & $-61.6 \%$ & $-84.9 \%$ & $-81.3 \%$ & $-79.8 \%$ \\
\hline Absolute & -1181 & -2940 & -9275 & $-13,396$ \\
\hline Mean & -295.3 & -735.0 & $-2,318.8$ & $-3,349.0$ \\
\hline SD & 130.5 & 976.4 & $1,473.5$ & $1,896.8$ \\
\hline \multicolumn{5}{|c|}{ DIFFERENCE BETWEEN POST-1 AND PRE-2 IN PERMANENT } \\
\hline \multicolumn{5}{|c|}{ SURGERY-RELATED LANGUAGE DEFICIT } \\
\hline Relative & $1,866.3 \%$ & $-22.9 \%$ & $-36.7 \%$ & $49.4 \%$ \\
\hline Absolute & 1493 & -112 & -469 & 912 \\
\hline \multicolumn{5}{|c|}{ DIFFERENCE BETWEEN POST-1 AND PRE-2 IN TRANSIENT } \\
\hline \multicolumn{5}{|c|}{ SURGERY-RELATED LANGUAGE DEFICITS } \\
\hline Relative & $-73.7 \%$ & $797.0 \%$ & $112.7 \%$ & $48.4 \%$ \\
\hline Absolute & -483 & 263 & 968 & 748 \\
\hline Mean & -161.0 & 87.7 & 322.7 & 249.3 \\
\hline SD & 252.8 & 90.9 & 179.3 & 298.8 \\
\hline \multicolumn{5}{|c|}{ DIFFERENCE BETWEEN PRE-1 AND POST-1 IN NEW } \\
\hline \multicolumn{5}{|c|}{ TUMOR-RELATED LANGUAGE DEFICITS } \\
\hline Relative & $-84.9 \%$ & $-61.1 \%$ & $-95.8 \%$ & $-89.5 \%$ \\
\hline Absolute & $-9,290$ & -933 & $-14,486$ & $-24,709$ \\
\hline Mean & $-3,096.7$ & -311.0 & $-4,828.7$ & $-8,236.3$ \\
\hline SD & $3,245.3$ & 444.8 & 4,332.2 & $6,231.1$ \\
\hline \multicolumn{5}{|c|}{ DIFFERENCE BETWEEN POST-1 AND PRE-2 IN NEW } \\
\hline \multicolumn{5}{|c|}{ TUMOR-RELATED LANGUAGE DEFICITS } \\
\hline Relative & $-59.5 \%$ & $-99.3 \%$ & $-94.8 \%$ & $-75.5 \%$ \\
\hline Absolute & -986 & -591 & -606 & $-2,183$ \\
\hline Mean & -328.7 & -197.0 & -202.0 & -727.7 \\
\hline SD & 726.2 & 272.3 & 102.5 & 973.6 \\
\hline \multicolumn{5}{|c|}{ DIFFERENCE BETWEEN PRE-1 AND POST-1 IN PATIENTS } \\
\hline \multicolumn{5}{|c|}{ WITHOUT NEW LANGUAGE DEFICIT } \\
\hline Relative & $134.3 \%$ & $55.5 \%$ & $125.0 \%$ & $124.8 \%$ \\
\hline Absolute & 975 & 61 & 3,719 & 4,755 \\
\hline Mean & 325.0 & 20.3 & 1239.7 & $1,585.0$ \\
\hline SD & 681.5 & 115.6 & $2,397.3$ & $3,024.9$ \\
\hline \multicolumn{5}{|c|}{ DIFFERENCE BETWEEN POST-1 AND PRE-2 IN PATIENTS } \\
\hline \multicolumn{5}{|c|}{ WITHOUT NEW LANGUAGE DEFICIT } \\
\hline Relative & $75.1 \%$ & $246.8 \%$ & $10.2 \%$ & $27.8 \%$ \\
\hline Absolute & 1,277 & 422 & 682 & 2,381 \\
\hline Mean & 425.7 & 140.7 & 227.3 & 793.7 \\
\hline SD & $1,184.0$ & 253.2 & $1,551.6$ & $2,495.2$ \\
\hline
\end{tabular}

The table shows the summarized changes of single white matter pathway fibers for the different subgroups of patients.

interdict the exclusive application of preoperative DTI FT data in neurosurgical cases, especially the possibility of revealing false negative results. Methodological innovations for the correction of DTI distortions and new algorithms for the optimization of image fusion address some of these issues. However, the presented approach of nrTMS-based DTI FT enables to give undirected DTI data a function-based starting and endpoint by adding ROIs based on a noninvasive mapping technique based on the same principle as the gold standard technique DES. Thereby, nrTMS-based DTI FT qualifies for long-term follow up examinations of cortical and subcortical functions and might contribute to the decision of re-operation, particularly in patients suffering from tumor residuals or recurrence of highly eloquent low grade gliomas. Additionally, the presented technique of single white matter pathway tracking might help basic researchers to assign higher brain functions to specific white matter pathways and subcortical networks.

\section{Limitations}

For the present study we used a deterministic DTI tractography algorithm. Pros and cons of deterministic vs. probabilistic algorithms and different diffusion models such as q-ball imaging and multiple others are controversially discussed (43, 44). Comparisons between different algorithms and imaging techniques showed that the tractography result is highly dependent on the respective mode (45-47). Hence, the application of only one tractography algorithm must be seen as a limitation of our study. In contrast, by using ROIs based on the preoperative mapping by nrTMS, the present study outlines the beneficial option of giving tractography results a functionbased starting and end point. Our presented standard protocol is integrated in the setup of our department. Here it must be highlighted that the feasibility with regard to the time that is required for the performance of tractography must be considered especially for its application in neurosurgery. Furthermore, the core of our results bases on relative changes of white matter language pathways and the correlation to the according status of language function. Although other tractography algorithms might have revealed different total numbers of fibers, the analysis of relations in the present study rules out a fundamental misinterpretation of data.

Changes in a functional network, particularly after the resection of gliomas, always base on a combination of cortical and subcortical adaptations. As shown by cortical language re-mappings by direct cortical stimulation during awake craniotomy, the cortical localization of language function changes over time after the resection of gliomas (32). However, for the present manuscript we focused on subcortical changes in dependence on the current status of language function. Particularly permanent language deficits after the resection of gliomas are mainly due to damaged white matter pathways. Based on the present results we were able to confirm this by DTI FT of single white matter pathways. We can exclude the impact of cortical changes between PRE- 1 and POST- 1 since we used the same cortical ROIs for these two DTI FTs. For the DTI FT at PRE2 we used new nrTMS language mapping results at PRE- 2 and the according DTI sequences at PRE-2 as described in the methods section. Cortical nrTMS language mapping data were used for the initial whole-language-network tractography. By the setting of additional anatomical ROIs for the single white matter pathway tracking of the FAT, the IFOF, and the SLF/AF, the algorithm is able to visualize definitely existing fibers exclusively. Thereby, the influence of cortical changes between these two examinations is reduced to a minimum through the special technique of single white matter pathway trackings we used for the present analysis. 
Finally, the cohort size of our pilot study with only 10 patients and the variability of time intervals between imagings and mappings is a limitation of our study. However, the present cohort enabled us to provide highly valuable tractographies of white matter language pathways in glioma patients including long-term follow up data and the positive correlation with the according status of language function.

\section{CONCLUSION}

By the present results we were able to show that nrTMSbased DTI FT of the IFOF, FAT, and SLF/AF mainly correlates with the according status of language function preoperatively,

\section{REFERENCES}

1. Sanai N, Mirzadeh Z, Berger MS. Functional outcome after language mapping for glioma resection. $N$ Engl J Med. (2008) 358:18-27. doi: 10.1056/NEJMoa067819

2. Ius T, Angelini E, Thiebaut De Schotten M, Mandonnet E, Duffau H. Evidence for potentials and limitations of brain plasticity using an atlas of functional resectability of WHO grade II gliomas: towards a "minimal common brain". Neuroimage (2011) 56:992-1000. doi: 10.1016/j.neuroimage.2011.03.022

3. De Witt Hamer PC, Hendriks EJ, Mandonnet E, Barkhof F, Zwinderman $\mathrm{AH}$, Duffau H. Resection probability maps for quality assessment of glioma surgery without brain location bias. PLoS ONE (2013) 8:e73353. doi: 10.1371/journal.pone.0073353

4. Henry RG, Berman JI, Nagarajan SS, Mukherjee P, Berger MS. Subcortical pathways serving cortical language sites: initial experience with diffusion tensor imaging fiber tracking combined with intraoperative language mapping. Neuroimage (2004) 21:616-22. doi: 10.1016/j.neuroimage.2003.09.047

5. Catani M, Thiebaut De Schotten M. A diffusion tensor imaging tractography atlas for virtual in vivo dissections. Cortex (2008) 44:1105-32. doi: 10.1016/j.cortex.2008.05.004

6. Sanai N, Berger MS. Intraoperative stimulation techniques for functional pathway preservation and glioma resection. Neurosurg Focus (2010) 28:E1. doi: $10.3171 / 2009.12$.FOCUS09266

7. Thiebaut De Schotten M, Ffytche DH, Bizzi A, Dell'acqua F, Allin M, Walshe $\mathrm{M}$, et al. Atlasing location, asymmetry and inter-subject variability of white matter tracts in the human brain with MR diffusion tractography. Neuroimage (2011) 54:49-59. doi: 10.1016/j.neuroimage.2010.07.055

8. Duffau H, Moritz-Gasser S, Mandonnet E. A re-examination of neural basis of language processing: proposal of a dynamic hodotopical model from data provided by brain stimulation mapping during picture naming. Brain Lang. (2014) 131:1-10. doi: 10.1016/j.bandl.2013.05.011

9. Chang EF, Raygor KP, Berger MS. Contemporary model of language organization: an overview for neurosurgeons. J Neurosurg. (2015) 122:250-61. doi: $10.3171 / 2014.10$.JNS132647

10. Moritz-Gasser S, Herbet G, Duffau H. Mapping the connectivity underlying multimodal (verbal and non-verbal) semantic processing: a brain electrostimulation study. Neuropsychologia (2013) 51:1814-22. doi: 10.1016/j.neuropsychologia.2013.06.007

11. Catani M, Dell'acqua F, Vergani F, Malik F, Hodge H, Roy P, et al. Short frontal lobe connections of the human brain. Cortex (2012) 48:273-91. doi: 10.1016/j.cortex.2011.12.001

12. Thiebaut De Schotten M, Dell'acqua F, Valabregue R, Catani M. Monkey to human comparative anatomy of the frontal lobe association tracts. Cortex (2012) 48:82-96. doi: 10.1016/j.cortex.2011.10.001

13. Fujii M, Maesawa S, Motomura K, Futamura M, Hayashi Y, Koba I, et al. Intraoperative subcortical mapping of a language-associated deep frontal tract connecting the superior frontal gyrus to Broca's area in the postoperatively, and at long-term follow up after the resection of left-sided perisylvian gliomas.

\section{AUTHOR CONTRIBUTIONS}

SI is responsible for data acquisition and handled the acquired data, performed statistical analyses, performed literature research, and drafted the manuscript. LE was responsible for data acquisition. AK was responsible for data acquisition. BM approved and corrected the final version of the manuscript. SK revised the manuscript, approved and corrected the final version, and is responsible for the original idea, the concept, design, data acquisition, and statistical analyses. All authors read and approved the final manuscript. dominant hemisphere of patients with glioma. J Neurosurg. (2015) 122:13906. doi: 10.3171/2014.10.JNS14945

14. Kinoshita M, De Champfleur NM, Deverdun J, Moritz-Gasser S, Herbet G, Duffau H. Role of fronto-striatal tract and frontal aslant tract in movement and speech: an axonal mapping study. Brain Struct Funct. (2015) 220:3399412. doi: $10.1007 / \mathrm{s} 00429-014-0863-0$

15. Picht T, Krieg SM, Sollmann N, Rosler J, Niraula B, Neuvonen T, et al. A comparison of language mapping by preoperative navigated transcranial magnetic stimulation and direct cortical stimulation during awake surgery. Neurosurgery (2013) 72:808-19. doi: 10.1227/NEU.0b013e31828 $89 \mathrm{e} 01$

16. Krieg SM, Sollmann N, Hauck T, Ille S, Meyer B, Ringel F. Repeated mapping of cortical language sites by preoperative navigated transcranial magnetic stimulation compared to repeated intraoperative DCS mapping in awake craniotomy. BMC Neurosci. (2014) 15:20. doi: 10.1186/1471-2202-15-20

17. Ille S, Sollmann N, Butenschoen VM, Meyer B, Ringel F, Krieg SM. Resection of highly language-eloquent brain lesions based purely on rTMS language mapping without awake surgery. Acta Neurochir. (2016) 158:226575. doi: 10.1007/s00701-016-2968-0

18. Sollmann N, Kubitscheck A, Maurer S, Ille S, Hauck T, Kirschke JS, et al. Preoperative language mapping by repetitive navigated transcranial magnetic stimulation and diffusion tensor imaging fiber tracking and their comparison to intraoperative stimulation. Neuroradiology (2016) 58:807-18. doi: 10.1007/s00234-016-1685-y

19. Pascual-Leone A, Bartres-Faz D, Keenan JP. Transcranial magnetic stimulation: studying the brain-behaviour relationship by induction of 'virtual lesions'. Philos Trans R Soc Lond B Biol Sci. (1999) 354:1229-38. doi: $10.1098 /$ rstb.1999.0476

20. Negwer C, Ille S, Hauck T, Sollmann N, Maurer S, Kirschke JS, et al. Visualization of subcortical language pathways by diffusion tensor imaging fiber tracking based on rTMS language mapping. Brain Imaging Behav. (2016) 11:899-914. doi: 10.1007/s11682-016-9563-0

21. Raffa G, Bahrend I, Schneider H, Faust K, Germano A, Vajkoczy P, et al A novel technique for region and linguistic specific nTMS-based DTI fiber tracking of language pathways in brain tumor patients. Front Neurosci. (2016) 10:552. doi: 10.3389/fnins.2016.00552

22. Rossini PM, Burke D, Chen R, Cohen LG, Daskalakis Z, Di Iorio R, et al. Noninvasive electrical and magnetic stimulation of the brain, spinal cord, roots and peripheral nerves: basic principles and procedures for routine clinical and research application. An updated report from an IFCN Committee. Clin Neurophysiol. (2015) 126:1071-107. doi: 10.1016/j.clinph.2015. 02.001

23. Krieg S, Lioumis P, Mäkelä J, Wilenus J, Karhu J, Hannula H, et al. Protocol for motor and language mapping by navigated TMS in patients and healthy volunteers; workshop report. Acta Neurochir. (2017) 159:1187-95. doi: 10.1007/s00701-017-3187-z

24. Huber WWD, Poeck K, Willmes K. Der aachener aphasie test-aufbau und überprüfung der konstruktion. Der Nervenarzt. (1980) 51:475-82. 
25. Negwer C, Beurskens E, Sollmann N, Maurer S, Ille S, Giglhuber K, et al. Loss of subcortical language pathways correlates with surgeryrelated aphasia in patients with brain tumor: an investigation via repetitive navigated transcranial magnetic stimulation-based diffusion tensor imaging fiber tracking. World Neurosurg. (2018) 111:e806-18. doi: 10.1016/j.wneu.2017.12.163

26. Ille S, Gempt J, Meyer B, Krieg SM. nTMS guidance of awake surgery for highly eloquent gliomas. Neurosurg Focus (2018) 45:V9. doi: 10.3171/2018.10.FocusVid.1873

27. Picht T, Kombos T, Gramm HJ, Brock M, Suess O. Multimodal protocol for awake craniotomy in language cortex tumour surgery. Acta Neurochir. (2006) 148:127-37. discussion: 137-28. doi: 10.1007/s00701-005-0706-0

28. Talacchi A, Santini B, Casagrande F, Alessandrini F, Zoccatelli G, Squintani GM. Awake surgery between art and science. Part I: clinical and operative settings. Funct Neurol. (2013) 28:205-21. doi: 10.11138/FNeur/2013.28.3.205

29. Talacchi A, Santini B, Casartelli M, Monti A, Capasso R, Miceli G. Awake surgery between art and science. Part II: language and cognitive mapping. Funct Neurol. (2013) 28:223-39. doi: 10.11138/FNeur/2013.28.3.223

30. Duffau $H$. The huge plastic potential of adult brain and the role of connectomics: new insights provided by serial mappings in glioma surgery. Cortex (2014) 58:325-37. doi: 10.1016/j.cortex.2013.08.005

31. Duffau H, Denvil D, Capelle L. Long term reshaping of language, sensory, and motor maps after glioma resection: a new parameter to integrate in the surgical strategy. J Neurol Neurosurg Psychiatry (2002) 72:511-6. doi: $10.1136 \% 2 F j n n p .72 .4 .511$

32. Southwell DG, Hervey-Jumper SL, Perry DW, Berger MS. Intraoperative mapping during repeat awake craniotomy reveals the functional plasticity of adult cortex. J Neurosurg. (2016) 124:1460-9. doi: 10.3171/2015.5.JNS1 42833

33. Fischer TM, Blazis DE, Priver NA, Carew TJ. Metaplasticity at identified inhibitory synapses in Aplysia. Nature (1997) 389:860-5. doi: 10.1038/39892

34. Duffau H. Brain plasticity: from pathophysiological mechanisms to therapeutic applications. J Clin Neurosci. (2006) 13:885-97. doi: 10.1016/j.jocn.2005.11.045

35. Kong NW, Gibb WR, Tate MC. Neuroplasticity: insights from patients harboring gliomas. Neural Plast. (2016) 2016:2365063. doi: $10.1155 / 2016 / 2365063$

36. Nunes MC, Roy NS, Keyoung HM, Goodman RR, Mckhann G. II, Jiang L, et al. Identification and isolation of multipotential neural progenitor cells from the subcortical white matter of the adult human brain. Nat Med. (2003) 9:439-47. doi: 10.1038/nm837

37. Duffau H. Does post-lesional subcortical plasticity exist in the human brain? Neurosci Res. (2009) 65:131-5. doi: 10.1016/j.neures.2009.07.002

38. Sollmann N, Meyer B, Krieg S. Implementing functional preoperative mapping in the clinical routine of a neurosurgical department. World Neurosur. (2017) 103:94-105. doi: 10.1016/j.wneu.2017. 03.114

39. Berman JI, Berger MS, Chung SW, Nagarajan SS, Henry RG. Accuracy of diffusion tensor magnetic resonance imaging tractography assessed using intraoperative subcortical stimulation mapping and magnetic source imaging. J Neurosurg. (2007) 107:488-94. doi: 10.3171/JNS-07/ $09 / 0488$
40. Bello L, Gambini A, Castellano A, Carrabba G, Acerbi F, Fava E, et al. Motor and language DTI fiber tracking combined with intraoperative subcortical mapping for surgical removal of gliomas. Neuroimage (2008) 39:369-82. doi: 10.1016/j.neuroimage.2007.08.031

41. Leclercq D, Duffau H, Delmaire C, Capelle L, Gatignol P, Ducros M, et al. Comparison of diffusion tensor imaging tractography of language tracts and intraoperative subcortical stimulations. J Neurosurg. (2010) 112:503-11. doi: 10.3171/2009.8.JNS09558

42. Duffau H. Diffusion tensor imaging is a research and educational tool, but not yet a clinical tool. World Neurosurg. (2013) 82:e43-5. doi: 10.1016/j.wneu.2013.08.054

43. Tuch DS, Reese TG, Wiegell MR, Wedeen VJ. Diffusion MRI of complex neural architecture. Neuron (2003) 40:885-95. doi: 10.1016/S0896-6273(03)00758-X

44. Dell'acqua F, Catani M. Structural human brain networks: hot topics in diffusion tractography. Curr Opin Neurol. (2012) 25:375-83. doi: 10.1097/WCO.0b013e328355d544

45. Bucci M, Mandelli ML, Berman JI, Amirbekian B, Nguyen C, Berger MS, et al. Quantifying diffusion MRI tractography of the corticospinal tract in brain tumors with deterministic and probabilistic methods. Neuroimage Clin. (2013) 3:361-8. doi: 10.1016/j.nicl.2013.08.008

46. Pujol S, Wells W, Pierpaoli C, Brun C, Gee J, Cheng G, et al. The DTI challenge: toward standardized evaluation of diffusion tensor imaging tractography for neurosurgery. J Neuroimag. (2015) 25:875-82. doi: $10.1111 /$ jon. 12283

47. Maier-Hein KH, Neher PF, Houde JC, Cote MA, Garyfallidis E, Zhong J, et al. The challenge of mapping the human connectome based on diffusion tractography. Nat Commun. (2017) 8:1349. doi: 10.1038/s41467-017-01285-x

Conflict of Interest Statement: BM received honoraria, consulting fees, and research grants from Medtronic (Meerbusch, Germany), Icotec ag (Altstätten, Switzerland), and Relievant Medsystems Inc., (Sunnyvale, CA, USA), honoraria, and research grants from Ulrich Medical (Ulm, Germany), honoraria and consulting fees from Spineart Deutschland GmbH (Frankfurt, Germany) and DePuy Synthes (West Chester, PA, USA), and royalties from Spineart Deutschland $\mathrm{GmbH}$ (Frankfurt, Germany). SK is consultant for Nexstim Plc (Helsinki, Finland) and Spineart Deutschland GmbH (Frankfurt, Germany) and received honoraria from Medtronic (Meerbusch, Germany) and Carl Zeiss Meditec (Oberkochen, Germany). SK and BM received research grants and are consultants for Brainlab AG (Munich, Germany).

The remaining authors declare that the research was conducted in the absence of any commercial or financial relationships that could be construed as a potential conflict of interest.

Copyright () 2018 Ille, Engel, Kelm, Meyer and Krieg. This is an open-access article distributed under the terms of the Creative Commons Attribution License (CC BY). The use, distribution or reproduction in other forums is permitted, provided the original author(s) and the copyright owner(s) are credited and that the original publication in this journal is cited, in accordance with accepted academic practice. No use, distribution or reproduction is permitted which does not comply with these terms. 